\title{
DESIGN AND ANALYSIS FOR MOTORS CONTROL OF A 4-DOF PARALLEL ROBOT
}

\author{
Tudor Catalin Apostolescuํ, Georgeta Ionascu ${ }^{2}$, Silviu Petrache ${ }^{3}$, Lucian Bogatu $^{4}$, Laurentiu Adrian Cartal ${ }^{5}$ \\ ${ }^{1}$ Faculty of Informatics, Titu Maiorescu University, Bucharest, Romania \\ 2,3,4,5 Faculty of Mechanical Engineering and Mechatronics, POLITEHNICA University of Bucharest, Romania \\ E-mail: ionascu georgeta@yahoo.com
}

\begin{abstract}
In this paper, the complex robotic structure of a new 4-DOF parallel robot is designed in a mechatronic concept that integrates mechanical and control aspects. The kinematic and dynamic analysis for the control of motors that act the robot's arms are presented. Delphi, Lab View and Solid Works softwares are used. The adopted constructive solution of the robot, designed in Solid Works, has been presented. Then, the transposition of equations which describe the forward and inverse kinematic model of the robot has been performed using the Lab View programming environment, a program that allows calling the servomotors' control board - NI 7344 acquisition board. The angles for the actuated arms of robot have been calculated based on a program developed in the Delphi environment that contains both models - the forward and the inverse one. In order to determine the motor load, a dynamic computation and a simulation have been achieved in Comos Motion from the Solid Works programming environment. Different results were extracted in the form of diagrams: the torque for the three servomotors acting the arms, the most loaded servomotor - the one that drives arm 1 and the variation of the angular velocity for the arm 1 . Finally, the robot prototype has been achieved and interesting conclusions regarding the analyzed robotic structures have been inferred.
\end{abstract}

Keywords: Mechatronic Design, Parallel Robot, Motion Control.

\section{Introduction}

At the moment, most industrial robots are made with tree structure, in this case each axis is positioned in series to the previous one, and therefore we can say that the axes are positioned in line to each other. The advantage of such a structure is its simplicity, being easily fabricated and controlled. The disadvantage of these structures is that unlike the parallel structures, are less rigid.

In case of parallel robots, the actuators are arranged parallel to each other and aim to orientation and positioning of a mobile platform on which there is placed the end-effector. The drive for this type of robot can be linear or rotary. The advantages of such a structure are: stiffness, it is a more rigid structure than in those in the series, which is due to closed kinematic structure; kinematics increasing, high speeds and accelerations.

The disadvantage of such a structure is its complexity.

Based on the Stewart-Gough platform architecture, a significant number of mechanical devices have been proposed [1, 2, 3]. In many industrial applications, the systems with 4 DOFs („Degrees of Freedom”) are sufficient: 3 translations to move the carried object from one point to the other and 1 rotation to adjust the orientation in its final location. Several research performed in this domain, particularly those by Clavel [4], have led to innovative architectures such as the famous Delta robot $[5,6]$.

In order to develop such a complex robotic structure, a mechatronic concept that integrates mechanical and control aspects has been considered. The design of a new 4-DOF parallel robot and, also, the kinematic and dynamic analysis for the control of motors that act the robot's arms are presented in this paper.

\section{Presentation of the Adopted Constructive Solution}

Usually a parallel 4-DOF robot is achieved by using a linear or rotary actuating system, a fixed part which forms the base of the robot, three parallelogram mechanisms for the parallel movement of a mobile plate or of a mobile platform that has the role of positioning the effector and the working object (WO).

It also needs an orientation system that can be achieved by using an actuation made by means of a servomotor which can be placed on the fixed plate or on the mobile one and an effector. 
Another problem in designing this type of parallel structure is the location of the effector action, or in other words, the location of the fourth degrees of freedom.

In order to design the robot there were chosen spherical joints, rotary motors using harmonic gearboxes and the end effector was posed on the robot mobile platform.

For the robot designing the Solid Works software has been used. The figures from below are captures representing the final assembly of the parallel robot structure with 4-DOF.

The components of the rigid or fixed robotic structure $(1-5)$ and of the mobile subassembly (6 13) are shown in Fig. 1, as follows: 1 - frame made from square bars; 2 - gusset plate; 3 - corner; 4 the robot base or fixed upper plate; 5 - fixing rods, which make the connection between the frame and the robot base; 6 - gear motors; 7 - robot arm; 8 passive joints, the spherical coupling model used being an open one in a constructive version called "bar head" (detailed in Fig. 2); 9 and 10 - bars or rods that form a parallelogram; 11 - the mobile platform or lower plate; 12 - the effector; in the proposed solution it consists of a suction cup; 13 WO.

To achieve the kinematics of the parallel robot with four degrees of freedom, it is necessary to use three similar subassemblies, each one consisting of a gear motor, arm, spherical joints and rods forming a parallelogram mechanism.

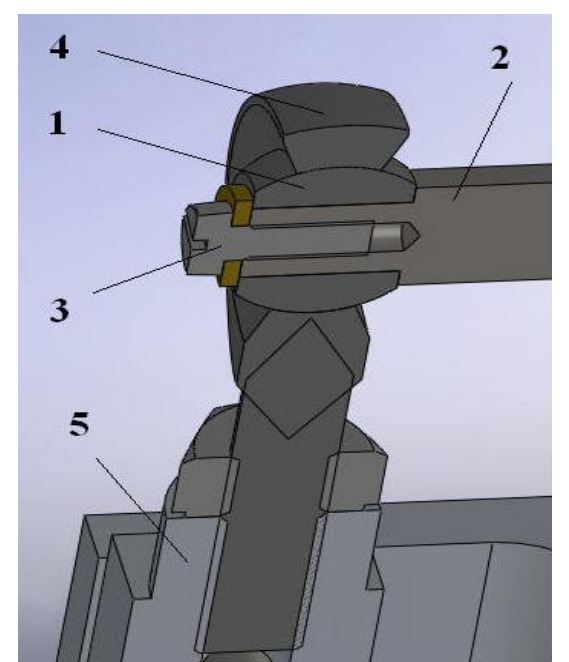

(a)

Figure 2: Type of joints:

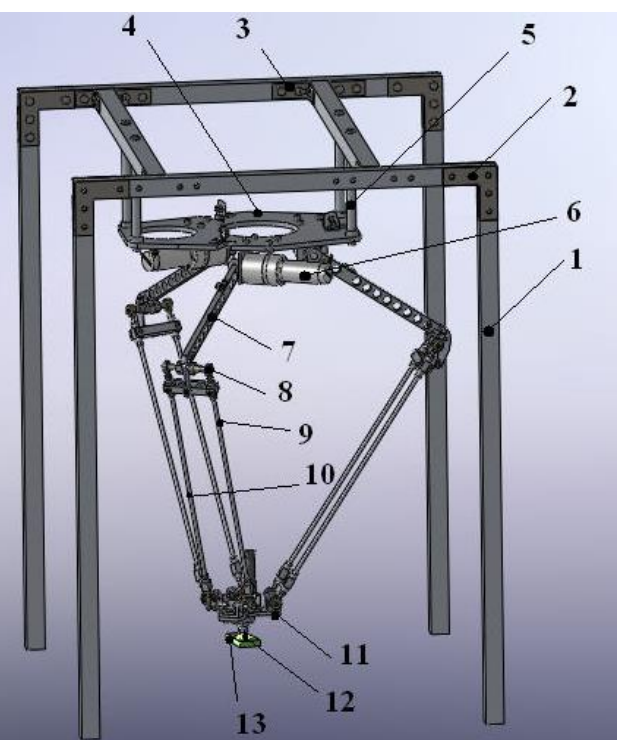

Figure 1: Assembly of the new 4-DOF parallel robot, represented in Solid Works.

The spherical joints can be replaced with two cylindrical joints with perpendicular axes. Fig. 2 shows the construction variant for the replacement of spherical joints with 2 cylindrical joints with perpendicular axes.

The spherical joint (Fig. 2 a) between the two elements 1 -arm and 2-rods with the center in 0 is replaced by two cylindrical couplings with $\mathrm{OX}$ and OY axes (Fig. 2 b).

To achieve the parallel robot with four degrees of freedom, we have chosen the spherical joint with its open version because of the precision and mobility considerations.

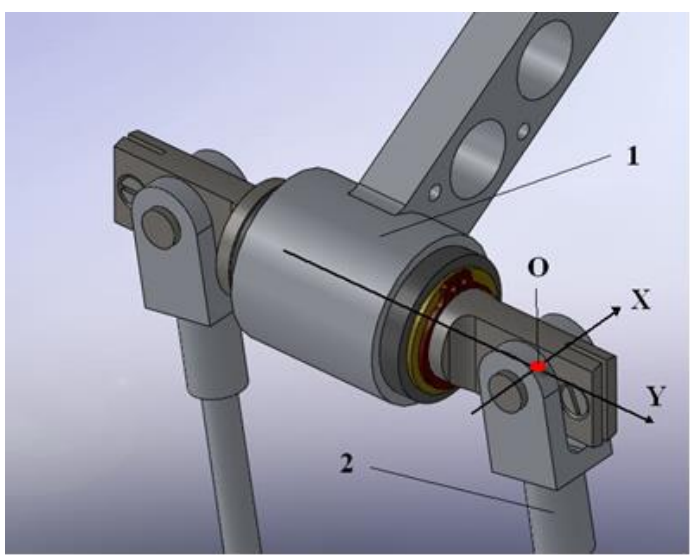

(b)

(a) the spherical joint using 1 - inner sphere, 2 - spindle, 3 - assembling screw, 4 - outer sphere, 5 - threaded bushing; (b) the cylindrical joint with 1 - arm, 2 - rods. 


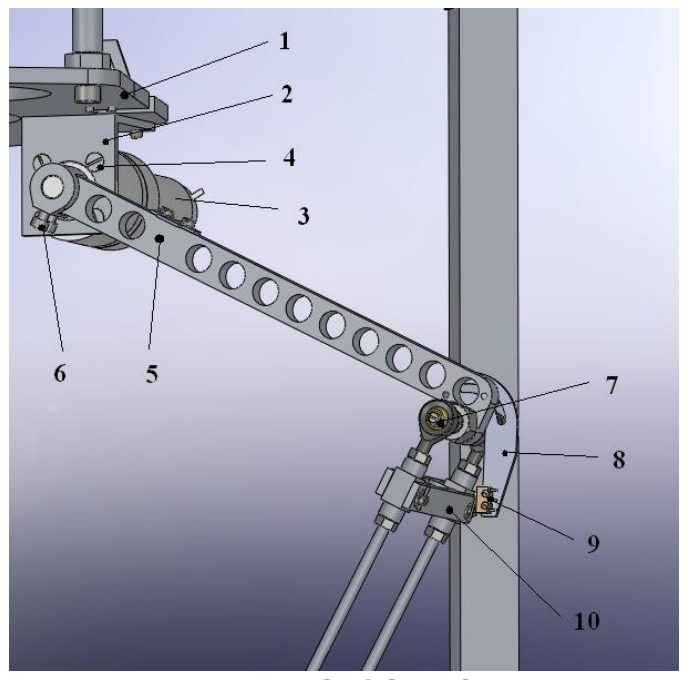

Figure 3: Detail of the robot arm.

Fig. 3 allows a better understanding of such a subassembly: 1 - the robot base or fixed upper plate; 2 - motor corner; 3 - gear motor; 4 - embedded screws for fixing the gear motor; 5 - robot arm; 6 clamping screws of the arm on the gear motor shaft; 7 - outer sphere of the spherical joint; 8 - corner of micro switch used to limit the stroke; 9 - end stroke (called "Forward") micro switch that blocks dangerous, unpredicted movement of the mobile platform; 10 - part used for preventing the rotation and, at the same time, for actuating the micro switch if it is necessary.

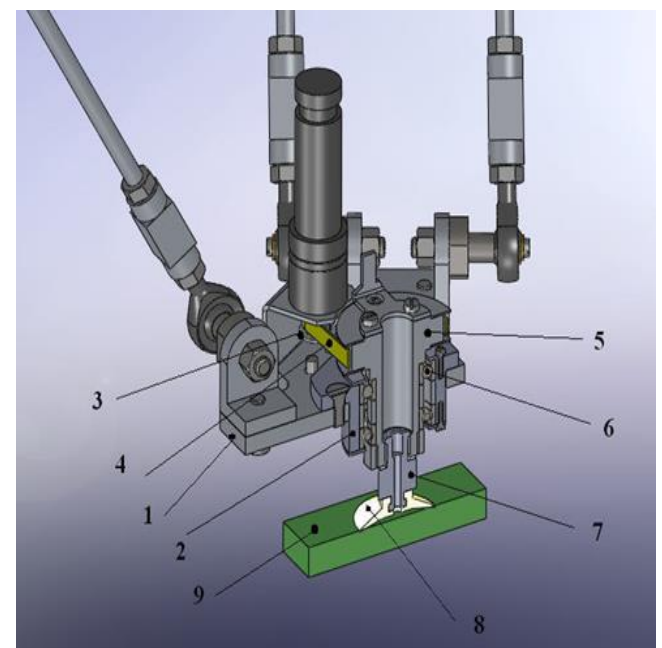

Figure 4: Detail of the mobile platform.

Fig. 4 shows a detail of the mobile platform and its component elements: 1 - the lower plate or mobile platform; 2 - orientation box; 3 - wheel belt mounted on the output shaft of the gear motor; 4 toothed belt; 5 - shaft - belt wheel; 6 - ball bearing; 7 - adapter for the suction cup; 8 - the effector represented by a suction cup; 9 - the working object (WO).
In Fig. 5 are detailed the following elements: 1 gear motor, 2 - toothed wheel driven by 5 - the toothed belt, 3 - mobile platform and 4 - effector, which in this case is a suction cup.

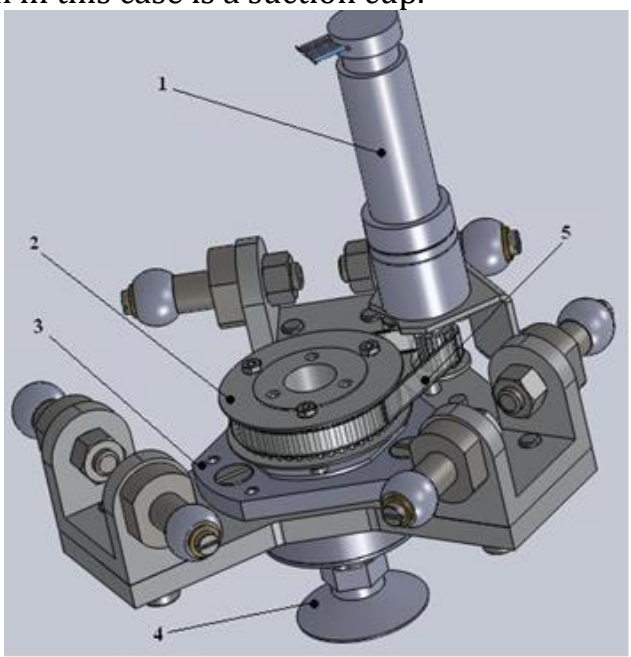

Figure 5: Sub-assembly represented by positioning of the effector actuation on the mobile platform

\section{The Computation Memoir}

\subsection{The computation of the forward and inverse geometric model}

The Delphi program does not have the ability to work with the NI 7344 servomotors control board. Therefore, was needed a similar program to that created in Delphi but written in a compatible programming environment.

The transposition of equations which describe the geometric forward and inverse model [7] was performed using the Lab View programming environment, a program that allows calling the servomotors control board - the NI 7344 acquisition board.

The purpose of this program is to be called as a sub function by Lab View programs designed to control the robot. Fig. 6 shows a simple way to call the sub-function.

There are used three controllers for introducing the Cartesian coordinates $\mathrm{x}_{0}, \mathrm{y}_{0}$ and $\mathrm{z}_{0}$ and also are employed three indicators for displaying the values of the resulting angles $\theta_{1}, \theta_{2}$ and $\theta_{3}$.

In a complex program, instead of the three controllers, are linked the values obtained by that program for the three Cartesian coordinates. Those three outputs are linked to the motion vector generation subprogram, and the resulting motion vector is sent to the servomotors motion control board. 

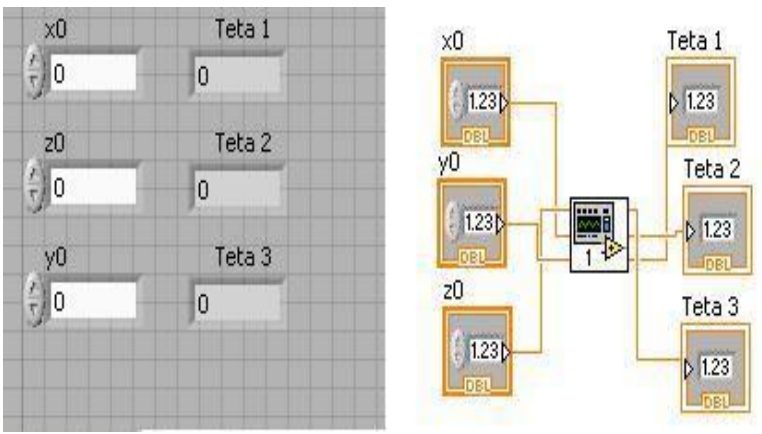

Figure 6: The call of the sub vi "sub vi program.vi". On the left is the control panel and on the right is the block diagram of the program.

Below is given a part of the computation program, which calculates the theta angle 3 . In the block diagram, the output variable is called "Delta 3".
Because the block diagram could not be included as a single figure, it was decomposed into 3 figures: Fig. 7, Fig. 8 and Fig. 9.

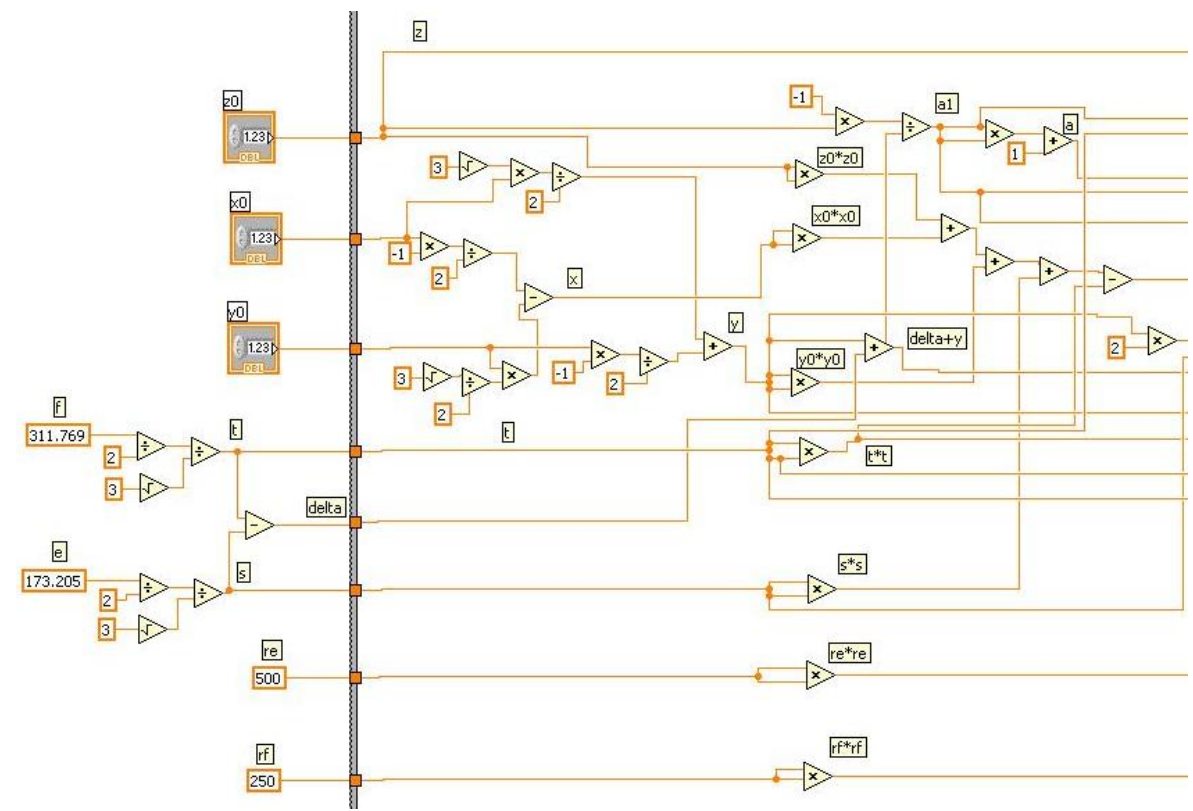

Figure 7: The block diagram for the sub vi which calculates the theta angle 3 - part 1.

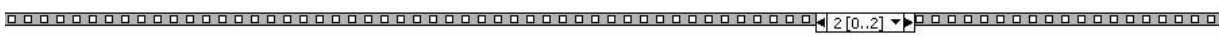

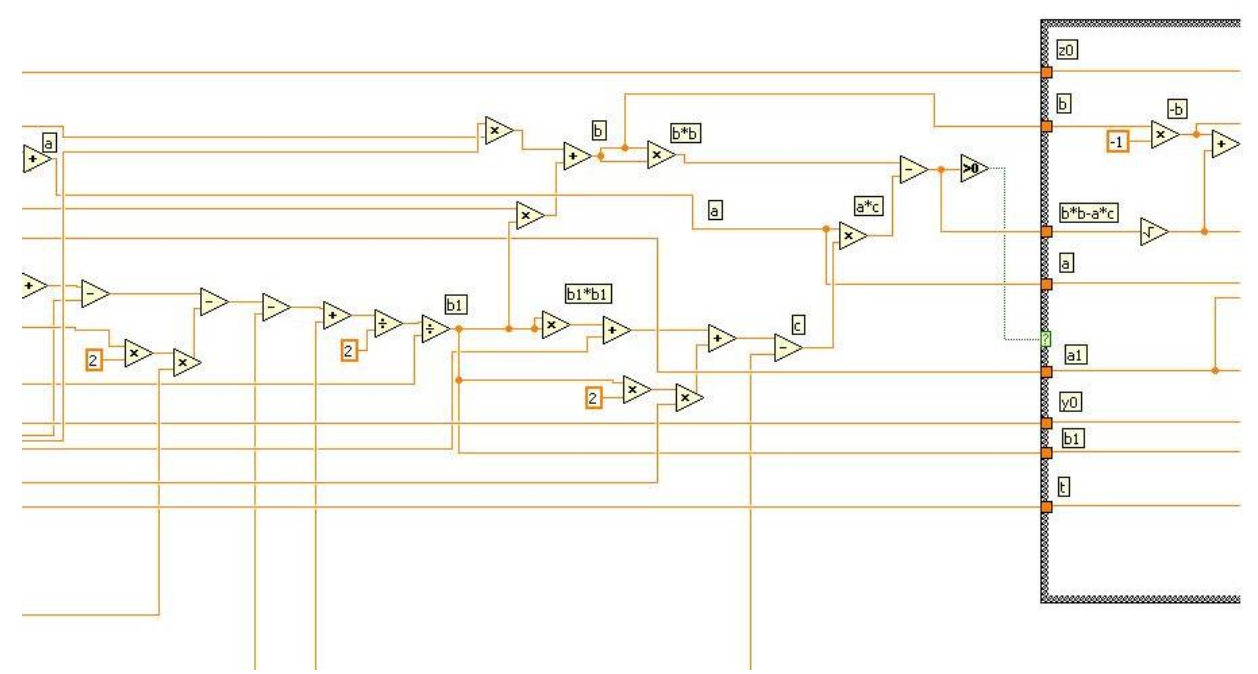

Figure 8: The block diagram for the sub vi which calculates the theta angle 3 - part 2. 


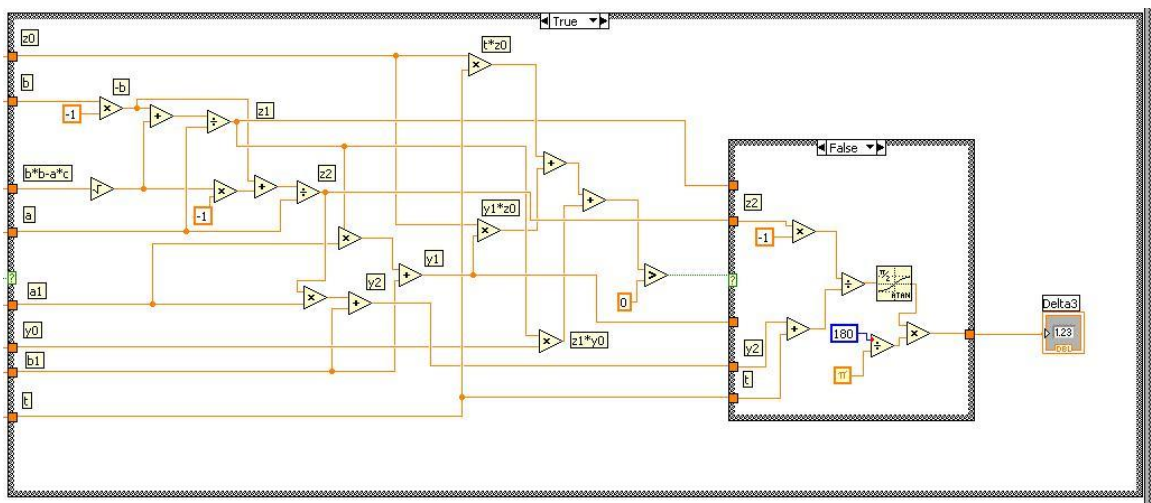

Figure 9: The block diagram for the sub vi which calculates the theta angle 3 - part 3.

\subsection{The computation of the robot arms' angles}

In order to calculation the arms' angles, we assume a linear trajectory between two points, initial $\mathrm{I}\left(\mathrm{x}_{\mathrm{I}}, \mathrm{y}_{\mathrm{I}}\right.$, $\mathrm{zI})$ and final $\mathrm{F}\left(\mathrm{xF}, \mathrm{yF}_{\mathrm{F}} \mathrm{zF}_{\mathrm{F}}\right)$ with the following coordinates (the position of the axes is according to Fig. 10): $\mathrm{x}_{\mathrm{I}}=-25 \mathrm{~mm} ; \mathrm{y}_{\mathrm{I}}=-25 \mathrm{~mm} ; \mathrm{zl}_{\mathrm{I}}=-550 \mathrm{~mm} ; \mathrm{x}_{\mathrm{F}}=$ $25 \mathrm{~mm} ; \mathrm{yF}_{\mathrm{F}}=25 \mathrm{~mm} ; \mathrm{zF}=-450 \mathrm{~mm}$.

The calculation of the $\theta$ angles for the actuated arms is based on the inverse geometric model. A "ProjectDelta2" program is developed in the Delphi environment that contains both models - the forward and the inverse one.

The values of the angles $\theta_{1}, \theta_{2}$ and $\theta_{3}$ are given in

Table 1. In the table are also mentioned the $\Delta \theta$ variations of the angles related to the values corresponding to the initial point I.

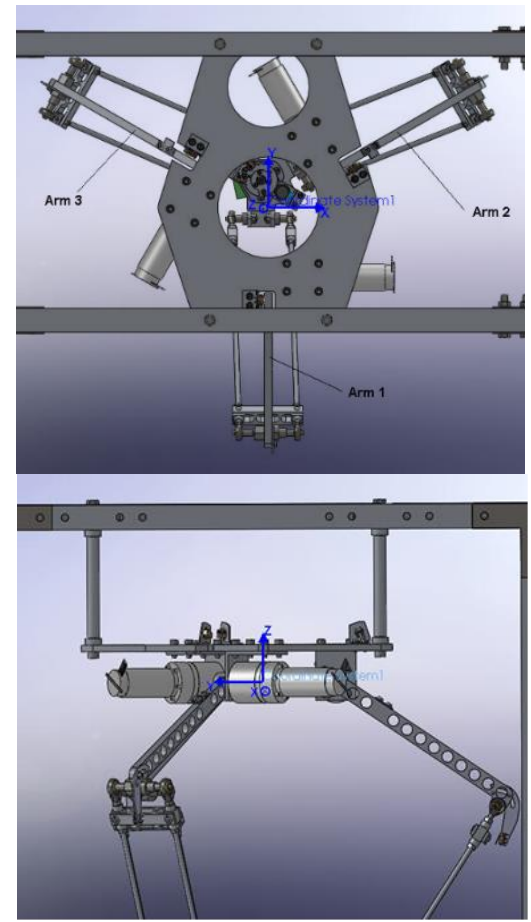

Figure 10: The position of the axes.
Table 1. The values for the angles $\theta_{1}, \theta_{2}$ and $\theta_{3}$.

\begin{tabular}{|l|l|l|l|}
\cline { 2 - 4 } \multicolumn{1}{c|}{} & Units & $\begin{array}{l}\text { Point I } \\
\text { (initial) }\end{array}$ & $\begin{array}{l}\text { Point } \mathrm{F} \\
\text { (final) }\end{array}$ \\
\hline $\mathrm{x}$ & $\mathrm{mm}$ & -25 & 25 \\
\hline $\mathrm{y}$ & $\mathrm{mm}$ & -25 & 25 \\
\hline $\mathrm{z}$ & $\mathrm{mm}$ & -550 & -450 \\
\hline$\theta_{1}$ & $\operatorname{deg}$ & $-26,5$ & $-13,2$ \\
\hline$\theta_{2}$ & $\operatorname{deg}$ & $-33,4$ & $-4,6$ \\
\hline$\theta_{3}$ & $\operatorname{deg}$ & $-28,4$ & $-10,9$ \\
\hline$\Delta \theta_{1}$ & $\operatorname{deg}$ & 0 & 13,3 \\
\hline$\Delta \theta_{2}$ & $\operatorname{deg}$ & 0 & 28,8 \\
\hline$\Delta \theta_{3}$ & $\operatorname{deg}$ & 0 & 17,5 \\
\hline
\end{tabular}

\subsection{Simulation of the arms' behavior}

To determine the engine load a dynamic computation and a simulation were performed in Comos Motion from the Solid Works programming environment.

In the simulation are inserted the servomotors (Rotary Motor) that actuate the arms - Fig. 11.

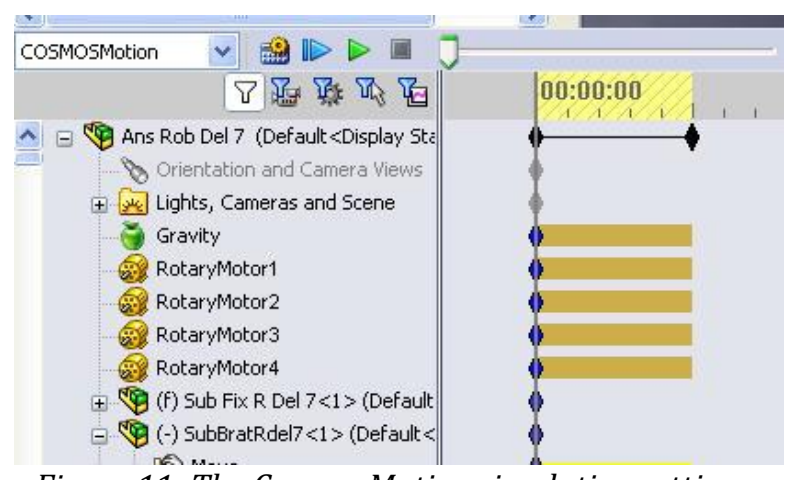

Figure 11: The Cosmos Motion simulation settings.

In Fig. 12 the MR1 servomotor (in RotaryMotor1 simulation) is shown to actuate the arm 1 of the structure. 


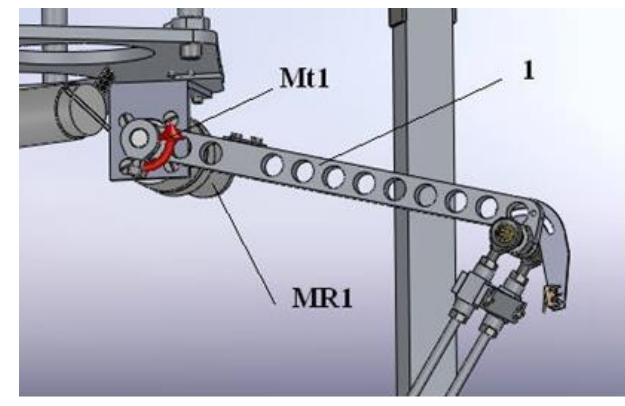

Figure 12: The Cosmos Motion simulation - the MR1 servomotor actuating the arm 1.

The motion kinematics is of the "step" type without acceleration leaps (fig.13). The angular acceleration curve $\varepsilon$ has no discontinuities and its variation is linear. Growth occurs during $\Delta t$. The angular velocity curve has parabolic variation, both on the acceleration zone and during the braking period.

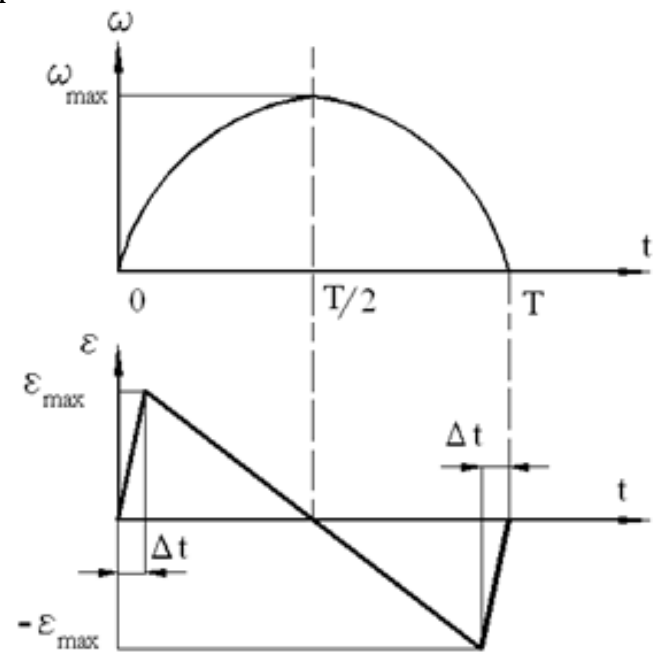

Figure 13: The Cosmos Motion simulation - the angular velocity and acceleration.

The settings of this motion for the simulation is shown in Fig. 14 where there are indicated the all the data for the kinematic of the RotaryMotor1 (MR1).

The step function includes the arguments: time, initial time (0s), initial angle (0D), final time (0.5s) and final angle (13.3D).

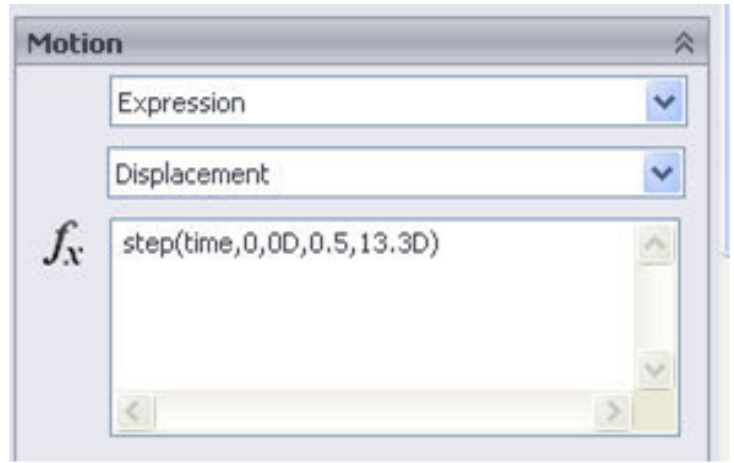

Figure 14: The Cosmos Motion simulation - the settings for the kinematic of MR1.
After completing the simulation, different results can be extracted in the form of diagrams.

The Fig. 15, Fig. 16 and Fig. 17 show the torque for the three servomotors acting on the arms: RotaryMotor1, RotaryMotor2 and RotaryMotor3.

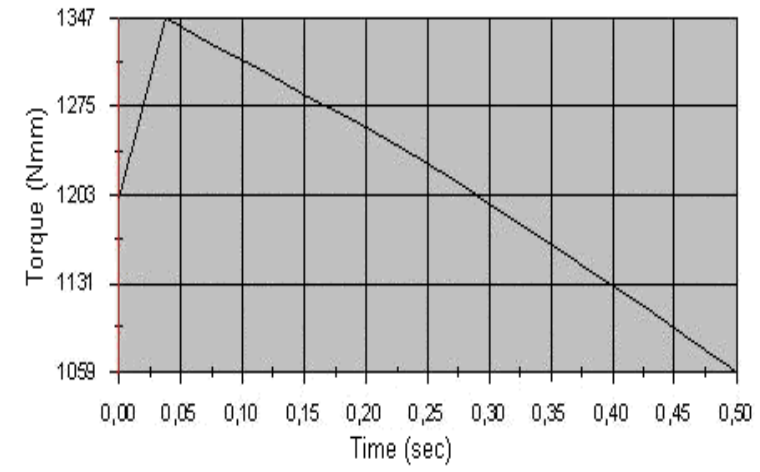

Figure 15: The Cosmos Motion simulation - the torque for MR1.

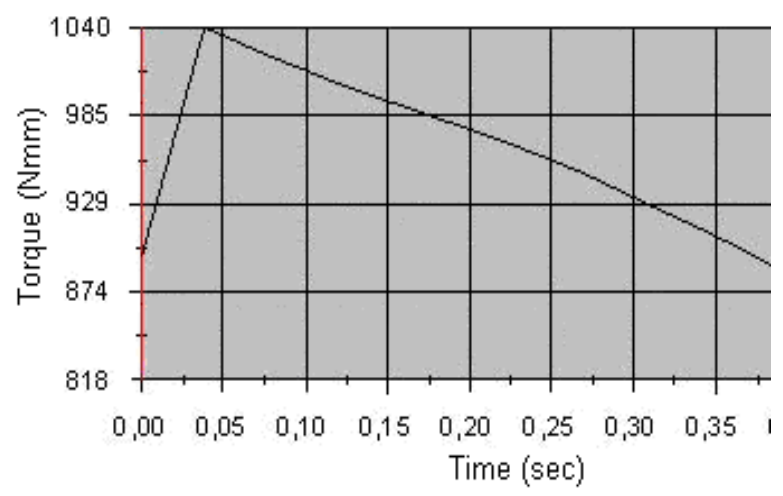

Figure 16: The Cosmos Motion simulation - the torque for $M R 2$.

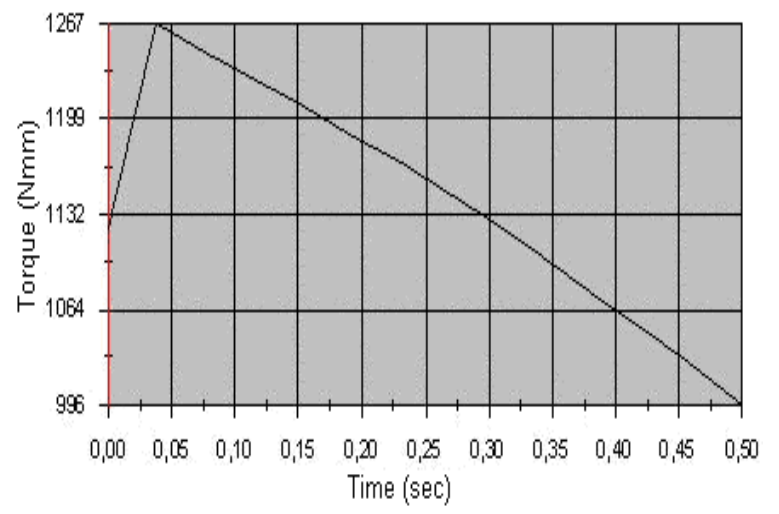

Figure 17: The Cosmos Motion simulation - the torque for MR3.

From chart analysis, the most loaded servomotor is the one that drives arm 1 (RotaryMotor1) requiring torque $\mathrm{Mt}=1347 \mathrm{Nmm}$.

The Fig. 18 shows the variation of the angular velocity of the arm 1. 


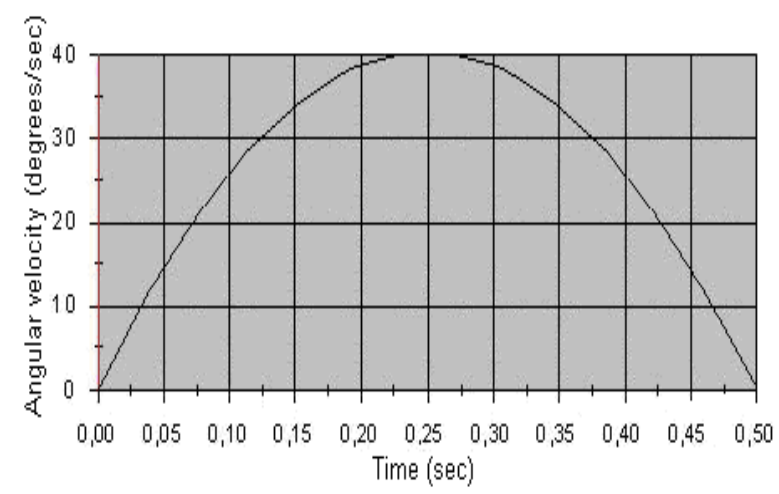

Figure 18: The Cosmos Motion simulation - the angular velocity of the arm 1 .

\section{Conclusions}

The feasibility of the original designing solutions for the new 4-DOF parallel robot has been demonstrated through achieving the prototype of this robot, Fig. 19.

Two kinds of experiments have been performed: manipulating the objects and image analysis.

The robot is able to detect new objects in the workspace and it can achieve object orientation even if this requires a rotation of more than $90^{\circ}$.

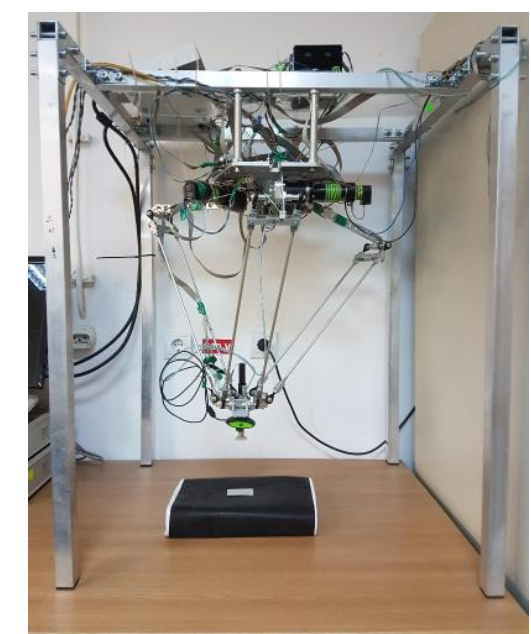

Figure 19: Prototype of the new 4-DOF parallel robot.

Taking into account the technical characteristics of the PMA-14A gear motor with a transmission ratio of 100 , it follows that the three gear motors acting the robot arms can reach a speed of little over $30 \mathrm{rpm}$ during continuous use, therefore the time of performing a cycle can significantly decrease to about 4 seconds, leading to the robot's ability to manipulate 15 objects per minute and a total of 900 objects per hour.

\section{References}

[1] Udrea, C., Alexandrescu, N., Panaitopol, H., Avram, M., Apostolescu, T.C.: The constructive bases of industrial robots (in Romanian), EdituraUniversitara Publishing House, Bucharest (2006).

[2] Merlet, J.P.: Parallel robots. 2nd edn. Springer, Dordrecht (2006).
[3] Siciliano, B., Khatib, O. (Eds.): Handbook of robotics. 2nd edn. Springer International Publishing (2016).

[4] Clavel, R.: Device for the movement and positioning of an element in space" U.S. Patent 4976582 (1990).

[5] Poppeová, V., Uríček, J., Bulej, V., Šindler P.: Delta robots - robots for high speed manipulation, Technical Gazette 18(3), 435-445 (2011).

[6] Stan, S.D., Manic, M., Szep, C., Balan, R.: Performance analysis of 3 DOF Delta parallel robot. In: HSI 2011 (4th International Conference of Human System Interaction), May 19-21, Keio University, Yokohama, Japan (2011).

[7] http://forums.trossenrobotics.com/tutorials/intr oduction-129/delta-robot-kinematics-3276/, last accessed 2016/05/30. 